\title{
Pneumatic tube system transport does not alter platelet function in optical and whole blood aggregometry, prothrombin time, activated partial thromboplastin time, platelet count and fibrinogen in patients on anti-platelet drug therapy
}

\author{
Dietmar Enko ${ }^{1,2}$, Harald Mangge ${ }^{2}$, Andreas Münch ${ }^{3}$, Tobias Niedrist ${ }^{2}$, Elisabeth Mahla ${ }^{3,4}$, Helfried Metzler ${ }^{3}$, Florian Prüller*2,4 \\ ${ }^{1}$ Institute of Clinical Chemistry and Laboratory Medicine, General Hospital Steyr, Steyr, Austria \\ ${ }^{2}$ Clinical Institute of Medical and Laboratory Diagnostics, Medical University Graz, Graz, Austria \\ ${ }^{3}$ Department of Anesthesiology and Intensive Care Medicine, Medical University Graz, Graz, Austria \\ ${ }^{4}$ Research Unit "Perioperative Platelet Function", Medical University of Graz, Graz, Austria \\ *Corresponding author: florian.prueller@medunigraz.at
}

\begin{abstract}
Introduction: The aim of this study was to assess pneumatic tube system (PTS) alteration on platelet function by the light transmission aggregometry (LTA) and whole blood aggregometry (WBA) method, and on the results of platelet count, prothrombin time (PT), activated partial thromboplastin time (APTT), and fibrinogen.

Materials and methods: Venous blood was collected into six $4.5 \mathrm{~mL}$ VACUETTE ${ }^{\circledR}$ 9NC coagulation sodium citrate $3.8 \%$ tubes (Greiner Bio-One International GmbH, Kremsmünster, Austria) from 49 intensive care unit (ICU) patients on dual anti-platelet therapy and immediately hand carried to the central laboratory. Blood samples were divided into 2 Groups: Group 1 samples $(N=49)$ underwent PTS $(4 \mathrm{~m} / \mathrm{s})$ transport from the central laboratory to the distant laboratory and back to the central laboratory, whereas Group 2 samples $(\mathrm{N}=49)$ were excluded from PTS forces. In both groups, LTA and WBA stimulated with collagen, adenosine-5'-diphosphate (ADP), arachidonic acid (AA) and thrombin-receptor-activated-peptide 6 (TRAP-6) as well as platelet count, PT, APTT, and fibrinogen were performed.

Results: No statistically significant differences were observed between blood samples with (Group 1) and without (Group 2) PTS transport (P values from 0.064 - 0.968). The AA-induced LTA (bias: $68.57 \%$ ) exceeded the bias acceptance limit of $\leq 25 \%$.

Conclusions: Blood sample transportation with computer controlled PTS in our hospital had no statistically significant effects on platelet aggregation determined in patients with anti-platelet therapy. Although AA induced LTA showed a significant bias, the diagnostic accuracy was not influenced.
\end{abstract}

Key words: platelets; platelet aggregation; platelet function tests; clinical laboratory services; preanalytical phase

\section{Introduction}

Platelets play a key role in primary hemostasis. The principal function is stopping hemorrhage and preventing blood loss, when vessel walls are injured (1). Platelets interacting with the exposed matrix adhere, become activated, and as a consequence bind further platelets to form a thrombus limited in size. Specific platelet receptors are involved in each of these phases (2).
Platelet function testing has become essential for identifying patients with platelet dysfunction and for monitoring modern anti-platelet therapy. In hemostasis laboratories, platelet aggregometry has been established one of the most widely used platelet function testing procedures (3). Light transmission aggregometry (LTA), which was developed in 1962 by Born and O'Brien, and imped- 
ance whole blood aggregometry (WBA) are the two main types of methodologies available (3-6).

Since platelets are sensitive to artificial manipulation, preanalytical variables may influence hemostasis testing in laboratories. The tourniquet application time is reported biasing platelet function testing by multiple electrode aggregometry (MEA) (Multiplate ${ }^{\circ}$ ) (7). Moreover, in clinical routine laboratories different vacuum tubes may represent a relevant source of variability on the determination of the mean platelet volume and platelet distribution width (8). Recently, one study concluded that physical exercise in cold water also represents a stress factor, which may cause increased platelet counts (9). Preanalytical variables are often outside the control of hemostasis laboratories and the leading causes of diagnostic error (10). Several crucial steps in the preanalytical phase, such as correct blood collection, blood sample handling, transport and storage of specimens are essential parts to get valid and timely laboratory test results (11).

Over the last years, in many hospitals pneumatic tube systems (PTS) have been established for blood sample transport between departments and outpatient clinics. PTS is considered an efficient and cost effective transport solution specially designed to handle automated blood specimen transport in routine and emergency settings. Nev- ertheless, blood samples transported by PTS are often subjected to high speed (up to $7.6 \mathrm{~m} / \mathrm{s}$ ) accompanied by rapid acceleration and deceleration causing shear stress (12). Changes in air pressure, vibrations and shaking of blood samples are considered to affect various laboratory parameters including coagulation assays (13).

In the literature, various studies have been performed on the effects of PTS on popular point-ofcare thromboelastometry $\left(\right.$ ROTEM $\left.^{\circ}\right)$ analysis. A study by Amann et al. including 20 healthy volunteers reported statistically significant changes ( $P$ values $<0.001)$ in multiple ROTEM $^{\circ}$ parameters transported by PTS (14). In contrast, Colucci et al. observed no statistically significant differences $(P$ values from 0.381 - 0.978) between pneumatic delivery and hand carried transport in 30 whole blood samples of healthy volunteers (15). While study results of PTS effects on ROTEM ${ }^{\oplus}$ analyses are discordant, only few data are available about platelet alteration after blood sample transportation with PTS on LTA and WBA, respectively. An overview on previously published original research articles investigating PTS effects on platelet function testing is given in Table 1.

The aim of the present study was to investigate the effect of PTS on the results of LTA, WBA, platelet count, prothrombin time (PT), activated partial thromboplastin time (APTT), and fibrinogen.

TABLE 1. Previously published original research articles investigating pneumatic tube system effects on platelet function testing

\begin{tabular}{|c|c|c|c|c|c|c|}
\hline Year & Author & Journal & Subjects, N & Methods & PTS effect & Reference \\
\hline 2004 & $\begin{array}{c}\text { Dyszkiewicz } \\
\text {-Korpanty et al. }\end{array}$ & JTH & 27 & $\begin{array}{c}\text { PFA-100 } \\
\text { Impedance WBA }\end{array}$ & $\begin{array}{c}\mathrm{CADP} \uparrow \\
\Omega \downarrow\end{array}$ & 18 \\
\hline 2005 & Walin et al. & CCLM & 28 & PFA-100 ${ }^{\mathrm{TM}}$ & No effect & 19 \\
\hline 2009 & Bollinger et al. & Platelets & 50 & Multiplate $^{\circ}$ & Aggregation $\downarrow$ & 20 \\
\hline 2010 & Hübner et al. & Clin Lab & 15 & $\begin{array}{l}\text { PFA-100 } \\
\text { Optical LTA }\end{array}$ & $\begin{array}{c}\text { CADP } \uparrow \\
\text { Aggregation } \downarrow\end{array}$ & 21 \\
\hline 2013 & Glas et al. & Platelets & 12 & $\begin{array}{l}\text { Multiplate }^{\circ} \\
\text { ROTEM }\end{array}$ & $\begin{array}{c}\text { Aggregation } \downarrow \\
\text { EXTEM CT } \uparrow\end{array}$ & 22 \\
\hline 2013 & Thalén et al. & Thromb Res & 58 & Multiplate ${ }^{\circ}$ & Aggregation $\downarrow$ & 23 \\
\hline
\end{tabular}

JTH - Journal of Thrombosis and Haemostasis. CCLM - Clinical Chemistry and Laboratory Medicine. Clin Lab - Clinical Laboratory. Thromb Res - Thrombosis Research. PFA - platelet function analyzer. CADP - collagen membrane coated with ADP. WBA - whole blood aggregometry. $\Omega$ - impedance. LTA - light transmission aggregometry. ROTEM - rotational thrombelastometry. EXTEM extrinsic rotational thrombelastometry. CT - clotting time. $\uparrow$ - increased. $\downarrow$ - decreased. 


\section{Materials and methods}

\section{Subjects}

This experimental study was conducted at the Medical University of Graz (Graz, Austria). The study period was from 01th January to 31th December 2008. A total of 49 patients from the Intensive Care Unit (ICU) of the Department of Anesthesiology and Intensive Care Medicine, who were on full dual anti-platelet drug therapy with $100 \mathrm{mg}$ acetyl-salicylate acid and $75 \mathrm{mg}$ clopidogrel daily, were included. Thirty-six (0.74) patients were male and $13(0.26)$ were female. The median age was 65 (range: $47-89$ ) years. This study was carried out in accordance with the latest version of the Declaration of Helsinki given by the World Medical Association. The ethical approval was provided by the Ethical Committee of the Medical University of Graz (Graz, Austria). All study subjects gave written informed consent for participation in the study.

\section{Blood sampling}

Blood sampling was done in the morning without obligatory fasting state. From all 49 study participants venous blood was collected into six $4.5 \mathrm{~mL}$ VACUETTE $^{\circledR}$ 9NC coagulation sodium citrate $3.8 \%$ tubes (Greiner Bio-One International $\mathrm{GmbH}$, Kremsmünster, Austria). Immediately after the venipuncture, all vacuum tubes ( 6 x 49) were gently inverted five times and hand carried by our laboratory personal to the core laboratory of our hospital. In the central laboratory blood samples were divided in Group 1 (3 x 49 tubes) and Group 2 ( 3 x 49 tubes) without delay. Group 1 blood samples were transported with the PTS from the central laboratory to the distant laboratory and returned again with the PTS from the distant laboratory to the central laboratory. Group 2 blood samples were excluded from PTS transport.

After transport all collected blood samples stored 30 minutes at room temperature. The original vacuum tubes 1 (Group 1 and Group 2) were gently inverted 5 times and $8 \times 450 \mu \mathrm{L}$ were pipetted in duplicates in each channel of a 4 channel Chronolog 700 Aggregometer (Chronolog Corporation, Havertown, Pennsylvania, USA) to perform impedance WBA. The original vacuum tubes 2 and 3 (Group 1 and Group 2) were centrifuged at $110 x$ $\mathrm{g}$ for 15 minutes at room temperature to obtain platelet rich plasma (PRP), using a GS-15 centrifuge (Beckman Coulter GmbH, Vienna, Austria). The PRP was removed from tubes 2 and 3 and mixed in 15 $\mathrm{mL}$ Eppendorf Conical Tubes ${ }^{\circ}$ (Eppendorf Austria $\mathrm{GmbH}$, Vienna, Austria) for further analysis. From the Eppendorf Conical Tubes ${ }^{\circledR}, 8 \times 450 \mu \mathrm{L}$ were pipetted in duplicates in each channel of a 4 channel Chronolog 700 Aggregometer (Chronolog Corporation, Havertown, Pennsylvania, USA) to perform LTA. Platelet count was performed from PRP on the Sysmex XE-2100 ${ }^{\mathrm{TM}}$ Automated Hematology System (Sysmex Austria GmbH, Vienna, Austria). Approximately $2.7 \mathrm{~mL}$ blood was left in each of the original vacuum tubes 2 and 3 (Group 1 and Group 2), which were centrifuged once more at $2800 \mathrm{xg}$ for $15 \mathrm{~min}$ utes at room temperature to obtain platelet poor plasma (PPP). PPP was used for PT, APTT and fibrinogen measurements and for blank value determinations of the aggregometer.

\section{Methods}

\section{Pneumatic tube system (PTS)}

Blood samples of Group 1 (3 x 49 tubes) were transported by a computer controlled PTS (Swisslog Rohrpostsysteme GmbH, Westerstede, Germany) using standard pneumatic tube carriers. One distance was about $500 \mathrm{~m}$ with a mean transit time of $2 \mathrm{~min}$. As a result Group 1 samples ( $3 \times 49$ tubes) were subjected a twice done acceleration and deceleration during transportation process with PTS, whereas Group 2 samples ( 3 x 49 tubes) were excluded from PTS transport.

After computer controlled acceleration an average velocity of $4 \mathrm{~m} / \mathrm{s}$ was used. Special receiving stations with controlled deceleration delivered softly the pneumatic tube carrier on special exit conveyor belts.

\section{Light transmission aggregometry (LTA)}

LTA was performed in duplicates using a 4 channel Chronolog 700 Aggregometer (Chronolog Corporation, Havertown, Pennsylvania, USA). After pipetting $450 \mu \mathrm{L}$ PRP in each channel, aggregation was induced using final concentrations of $2 \mu \mathrm{g} / \mathrm{mL}$ col- 
lagen (Chronolog Corporation, Havertown, Pennsylvania, USA), $10 \mu \mathrm{mol} / \mathrm{L}$ adenosine 5'-diphosphate (ADP) (Sigma-Aldrich Handels $\mathrm{GmbH}$, Vienna, Austria), $0.5 \mathrm{mmol} / \mathrm{L}$ arachidonic acid (AA) (Chronolog Corporation, Havertown, Pennsylvania, USA), and $40 \mu \mathrm{mol} / \mathrm{L}$ thrombin receptor-activated peptide 6 (TRAP-6) (Bachem Distribution Services $\mathrm{GmbH}$, Weil/Rhein, Germany). Light transmission (\% aggregation and area under the curve (AUC)) was measured in Group 1 and 2 and compared statistically. A maximal amplitude of $<5 \%$ and an AUC of $<75$ were interpreted as total inhibition of platelet function depending on the above mentioned platelet aggregation inducing reagents. Daily quality control (QC) measurements were performed with normal plasma of healthy volunteers. Coefficients of variation (CV) for light transmission $\%$ and AUC were as follows: collagen - 4.01 and 3.18\%, respectively; ADP - 3.38 and $6.53 \%$, respectively; $A A-2.19$ and $4.94 \%$, respectively; TRAP - 4.14 and $2.5 \%$, respectively.

\section{Impedance whole blood aggregometry (WBA)}

Impedance WBA was performed in duplicates using a 4 channel Chronolog 700 Aggregometer (Chronolog Corporation, Havertown, Pennsylvania, USA). After pipetting $450 \mu \mathrm{L}$ whole blood in each channel, aggregation was induced with the same agents and final concentrations as described above. The impedance ( $\Omega$ and AUC) was measured in Group 1 and 2 samples and compared statistically. A maximal impedance of $<0.5 \Omega$ and an AUC of $<5$ and were interpreted as total inhibition of platelet function depending on the above mentioned platelet aggregation inducing reagents. Daily QC measurements were performed with normal plasma of healthy volunteers. Coefficients of variation for impedance $\Omega$ and AUC were as follows: collagen 4.06 and $10.9 \%$, respectively; ADP - 13.47 and $12.38 \%$, respectively; $A A-13.72$ and $8.72 \%$, respectively; TRAP - 12.06 and $10.52 \%$, respectively.

\section{Coagulation parameters}

The number of platelets was determined on the Sysmex XE-2100 TM Automated Hematology System (Sysmex Austria GmbH, Vienna, Austria). The PT (Thromborel ${ }^{\circ} S$ reagent, Siemens Healthcare Diagnostics GmbH, Vienna, Austria), APTT (Pathrom- tin ${ }^{\circledR} \mathrm{L}$ reagent, Siemens Healthcare Diagnostics $\mathrm{GmbH}$, Vienna, Austria), and fibrinogen (Multifibren $^{\circ} \mathrm{U}$ reagent, Siemens Healthcare Diagnostics $\mathrm{GmbH}$, Vienna, Austria) were measured on the Siemens/Dade Behring BCS XP Analyzer Automated Coagulation System (Siemens Healthcare Diagnostics GmbH, Vienna, Austria). Daily QC measurements were performed with commercially available control material within the normal and pathological ranges (Control Plasma N and $\mathrm{P}$ (Siemens Healthcare Diagnostics GmbH, Vienna, Austria): PT 73.0 $109.0 \%$ and $32-48 \%$; APTT $28.0-38.0 \mathrm{~s}$ and $70-$ $105 \mathrm{~s}$; fibrinogen $2.2-3.2 \mathrm{~g} / \mathrm{L}$ and $0.6-1.4 \mathrm{~g} / \mathrm{L}$, respectively; e-CHECK $(\mathrm{XE})$ (Sysmex Austria $\mathrm{GmbH}$ ): platelet counts $195-240 \mathrm{G} / \mathrm{L}, 30-75 \mathrm{G} / \mathrm{L}$ and 480 $580 \mathrm{G} / \mathrm{L})$. Inter-assay precision was calculated with results of minimum 20 consecutive days. One tenfold measurement was performed at one day to determine the intra-assay precision. The intra- and inter-assay CV were as follows: for PT 1.71 and $3.26 \%$, respectively; for PT-INR 2.43 and 3.24\%, respectively; for APTT 2.31 and $2.83 \%$, respectively; for fibrinogen 1.23 and $2.87 \%$, respectively; and for platelet count 1.23 and $3.4 \%$, respectively.

\section{Statistical analysis}

The distribution of data was calculated with the Kolmogorov-Smirnov test. The non-parametric Wilcoxon test was used for comparisons of parameters between Group 1 and Group 2. Not normally distributed data were described in medians (Q1 - Q3). A P-value $<0.05$ was considered statistically significant. Bias calculation was performed and compared with available acceptance criteria in accordance with the Milano hierarchy (16). The calculated biases were compared to the acceptance limits based on the Clinical Laboratory Improvement Amendments (CLIA) for analytical quality (17). SPSS Statistics for Windows version 22.0 (IBM SPSS Inc., Chicago, Illinois, USA) was used for statistical analysis.

\section{Results}

The results of platelet function testing with LTA and WBA, and the coagulation parameters (i.e., platelet count, PT, APTT, and fibrinogen) between blood samples of Group $1(\mathrm{~N}=49)$ and Group 2 (N 
=49) and the mean bias are shown in Table 2. All parameters investigated in the present study were not normally distributed and are presented in medians (Q1 - Q3). No statistically significant differences were observed between blood samples transported with (Group 1) and without PTS (Group 2) (P values from $0.064-0.968$ ).

\section{Discussion}

To the best of our knowledge, this is the first study reporting data about PTS effects on both, optical aggregometry as well as impedance aggregrometry measurements in patients on anti-platelet drug therapy. The results referred only to patients on dual acetyl-salicylate acid and clopidogrel therapy

TABLE 2. Light transmission, impedance and coagulation parameters

\begin{tabular}{|c|c|c|c|c|c|c|}
\hline LTA & & $\begin{array}{l}\text { Group } 1 \\
(N=49)\end{array}$ & $\begin{array}{l}\text { Group } 2 \\
(N=49)\end{array}$ & P value & Bias (\%) & $\begin{array}{c}\text { Bias criteria } \\
(\%)\end{array}$ \\
\hline \multirow[t]{2}{*}{ Collagen-induced } & $\%$ & $28.0(16.8-47.0)$ & $28.5(14.0-50.0)$ & 0.592 & 1.75 & $\leq 25$ \\
\hline & AUC & $222.2(112.1-337.1)$ & $227.7(99.3-376.4)$ & 0.682 & 2.42 & $\leq 25$ \\
\hline \multirow[t]{2}{*}{ ADP-induced } & $\%$ & $33.0(24.0-40.8)$ & $35.5(21.0-43.0)$ & 0.406 & 7.04 & $\leq 25$ \\
\hline & AUC & $280.8(138.6-338.6)$ & $279.9(127.2-362.4)$ & 0.568 & 0.32 & $\leq 25$ \\
\hline \multirow[t]{2}{*}{ AA-induced } & $\%$ & $2.0(1.0-11.0)$ & $2.0(1.0-16.3)$ & 0.741 & 0.0 & $\leq 25$ \\
\hline & AUC & $23.6(0.25-51.35)$ & $14.0(0.0-52.5)$ & 0.064 & 68.57 & $\leq 25$ \\
\hline \multirow[t]{2}{*}{ TRAP-6-induced } & $\%$ & $51.0(35.0-59.0)$ & $52.0(43.5-62.0)$ & 0.141 & 1.92 & $\leq 25$ \\
\hline & AUC & $441.6(290.6-512.8)$ & $438.6(348.3-521.4)$ & 0.915 & 0.68 & $\leq 25$ \\
\hline \multicolumn{7}{|l|}{ WBA } \\
\hline \multirow[t]{2}{*}{ Collagen-induced } & $\Omega$ & $8.0(5.0-11.0)$ & $8.0(4.0-12.0)$ & 0.444 & 0.0 & $\leq 25$ \\
\hline & AUC & $44.8(18.8-66.1)$ & $40.1(18.6-74.9)$ & 0.165 & 11.72 & $\leq 25$ \\
\hline \multirow[t]{2}{*}{ ADP-induced } & $\Omega$ & $2.0(0.0-5.3)$ & $2.5(1.0-7.0)$ & 0.180 & 20.0 & $\leq 25$ \\
\hline & AUC & $8.55(0.0-31.6)$ & $11.0(0.0-42.5)$ & 0.361 & 22.27 & $\leq 25$ \\
\hline \multirow[t]{2}{*}{ AA-induced } & $\Omega$ & $0.0(0.0-0.0)$ & $0.0(0.0-0.0)$ & 0.549 & 0.0 & $\leq 25$ \\
\hline & AUC & $0.0(0.0-0.0)$ & $0.0(0.0-0.0)$ & 0.286 & 0.0 & $\leq 25$ \\
\hline \multirow[t]{2}{*}{ TRAP-6-induced } & $\Omega$ & $3.0(1.0-9.0)$ & $3.0(1.0-9.0)$ & 0.733 & 0.0 & $\leq 25$ \\
\hline & AUC & $22.9(5.6-70.7)$ & $25.2(2.1-65.4)$ & 0.266 & 9.13 & $\leq 25$ \\
\hline \multicolumn{7}{|c|}{ Coagulation parameters } \\
\hline \multirow{2}{*}{$\begin{array}{l}\text { Platelets (WB) } \\
\text { Platelets (PRP) }\end{array}$} & $\times 10^{9} / \mathrm{L}$ & $94.0(66.0-142.0)$ & $94.0(66.0-139.0)$ & 0.418 & 0.0 & $\leq 25$ \\
\hline & $\times 10^{9} / \mathrm{L}$ & $175.0(114.0-267.0)$ & $170.0(123.0-267.0)$ & 0.176 & 2.94 & $\leq 25$ \\
\hline \multirow[t]{2}{*}{ PT } & INR & $1.1(1.0-1.3)$ & $1.1(1.0-1.3)$ & 0.968 & 0.0 & $\leq 15$ \\
\hline & $\%$ & $91.0(72.0-107.0)$ & $92.0(75.0-107.0)$ & 0.909 & 1.09 & $\leq 15$ \\
\hline APTT & s & $42.4(38.3-49.8)$ & $43.5(38.3-49.7)$ & 0.426 & 2.53 & $\leq 15$ \\
\hline Fibrinogen & $g / L$ & $6.3(3.6-7.9)$ & $6.1(3.6-8.0)$ & 0.726 & 3.30 & $\leq 20$ \\
\hline
\end{tabular}

LTA - light transmission aggregometry. WBA - whole blood aggregometry. $\Omega$ - impedance. Group 1 - blood samples transported with pneumatic tube system (PTS). Group 2 - blood samples transported without PTS. ADP - adenosine diphosphate. AA arachidonic acid. TRAP-6 - thrombin receptor-activated peptide 6. AUC - area under the curve. WB - whole blood. PRP - platelet rich plasma. PT - prothrombin time. INR - international normalized ratio. APTT - activated partial thromboplastin time. Differences between Group 1 and Group 2 were evaluated using the Wilcoxon test. Data are presented as medians (Q1 - Q3). P < 0.05 was considered statistically significant. Bias criteria according to reference 17. 
and not on healthy individuals. Overall 49 study participants were included in this experimental study.

In comparison, previously published studies on the subject of PTS effects on platelet aggregation tests were designed with 12 to 58 subjects $(18-23)$ (Table 1). Two studies, comprising 58 and 50 individuals, observed significant influence on impedance WBA with a Multiplate analyzer $(20,23)$. In both studies, detailed information about speed of sample transport and other characteristics of the PTS, that may strongly affect pre-analytical quality are lacking. Reducing speed is considered to avoid pre-analytical alteration of blood samples (24). However, different PTS configurations could be a potential reason for various results and observations of published data.

In the present study both methodologies, namely LTA as well as WBA were performed to study PTS effects on platelet function. These methods are usually performed in specialized clinical hemostasis laboratories. Beside these traditional tests, previously published studies about PTS effects on platelet function used especially modern commercially available point-of-care testing methods, such as the multichannel WBA with Multiplate system or the relatively simple PFA- $100^{\mathrm{TM}}$ technology, measuring platelet aggregation as a function of the time it takes to occlude the aperture (25).

Poor correlation among different platelet function testing modalities may be one limitation in comparison and clinical interpretation of different study results. Moreover, different agonists are used to induce platelet aggregation. Herein we used the aggregation agonists collagen $(2 \mu \mathrm{g} / \mathrm{mL})$, ADP $(10 \mu \mathrm{mol} / \mathrm{L}), A A(0.5 \mathrm{mmol} / \mathrm{L})$, and TRAP-6 (40 $\mu \mathrm{mol} / \mathrm{L}$ ) with final concentrations as recommended by the manufacturer. In comparison Dyszkiewicz-Korpanty et al. recruited 27 healthy volunteers and used collagen $(2 \mu \mathrm{g} / \mathrm{mL})$, ADP $(10 \mu \mathrm{mol} / \mathrm{L})$ and $A A(0.5 \mathrm{mmol} / \mathrm{L})$ to perform impedance WBA (18), whereas Hübner et al. recruited 15 healthy subjects and used collagen $(2 \mu \mathrm{g} / \mathrm{mL}$ and $5 \mu \mathrm{g} /$ $\mathrm{mL}), \operatorname{ADP}(10 \mu \mathrm{mol} / \mathrm{L})$ and ristocetin $(1.25 \mathrm{~g} / \mathrm{L})$ to perform optical LTA (20). One previously published study about PTS effects on the Multiplate system was performed with 58 individuals (32 healthy individuals, 14 patients with cardiovascular disease treated with aspirin and/or clopidogrel, 8 patients from an ICU, and 4 patients from a coagulation clinic) using final concentrations of $0.0032 \mu \mathrm{g}$ collagen, $0.0065 \mu \mathrm{mol}$ ADP, $0.4839 \mu \mathrm{mol} A \mathrm{~A}, 0.3076$ $\mu \mathrm{g}$ ristocetin and $0.0322 \mu \mathrm{mol}$ TRAP in each corresponding well (23). Another study on the Multiplate ${ }^{\circ}$ system evaluated 50 patients with acetyl-salicylate acid therapy using $A A(0.5 \mathrm{mmol} / \mathrm{L})$ and TRAP (32 $\mu \mathrm{mol} / \mathrm{L}$ ) only (20).

Considering these various study designs performed with different agonists and concentrations, the major limitation of data comparison is the lack of standardization in platelet function testing. Widespread accepted uniform guidelines on how laboratories should perform clinical testing for disorders of platelet function are still not available $(26,27)$. Results of two surveys of the North American Specialized Coagulation Laboratory Association (NASCOLA) demonstrated that agonist concentrations varied widely and that various methods were used to obtain reference intervals for platelet aggregation testing (26). Although LTA is considered to be the "gold standard" for in vitro platelet function testing in both clinical and research laboratories, no evidence-based guidelines for the performance and interpretation of studies with this technique are established yet $(28,29)$. Moreover no proper commercial controls are available for the different platelet function assays to examine the validity of the test results.

In the present study the mean bias of the investigated parameters between blood samples transported with (Group 1) and without PTS (Group 2) ranged from 0.0 to $68.57 \%$. Compared to the available acceptance criteria in accordance with the Milano hierarchy $(16,17)$, analytical performance evaluation studies may be influenced by the measurement quality, the actual test method used, and the investigated study population. Therefore standardized platelet function testing procedures with commercially available QC material for LTA and WBA is required in order to improve analytical quality and reproducibility. 
The major limitation of this study is that healthy subjects, who were not on anti-platelet therapy, were not included. We cannot omit a protective effect on acetyl-salicylate acid and clopidogrel during transportation. Nevertheless, the added value of this study is that potential PTS effects were investigated on platelet function testing with the LTA as well as the WBA method.

In conclusion, blood sample transportation with soft motion computer controlled PTS, as installed in our hospital, had no statistically significant effects on platelet aggregation measured with optical and impedance aggregometry in patients with

\section{References}

1. Broos K, De Meyer SF, Feys HB, Vanhoorelbeke K, Deckmyn H. Blood platelet biochemistry. Thromb Res 2012;129:245-9. https://doi.org/10.1016/j.thromres.2011.11.002.

2. Clemetson KJ. Platelets and primary haemostasis. Thromb Res 2012;129:220-4. https://doi.org/10.1016/j.thromres.2011. 11.036.

3. Favaloro EJ, Lippi G, Franchini M. Contemporary platelet function testing. Clin Chem Lab Med 2010;48:579-98. https://doi.org/10.1515/CCLM.2010.121.

4. Born GV. Aggregation of blood platelets by adenosine diphosphate and its reversal. Nature 1962;194:927-9. https:// doi.org/10.1038/194927b0.

5. O'Brien JR. The adhesiveness of native platelets and its prevention. J Clin Pathol 1961;14:140-9. https://doi. org/10.1136/jcp.14.2.140.

6. Cardinal DC, Flower RJ. The study of platelet aggregation in whole blood [proceedings]. Br J Pharmacol 1979;66:94P95P.

7. Lima-Oliveira G, Lippi G, Salvagno GL, Gaino S, Poli G, Gelati $M$, et al. Venous stasis and whole blood platelet aggregometry: a question of data reliability and patient safety. Blood Coagul Fibrinolysis 2015;26:665-8. https://doi. org/10.1097/MBC.0000000000000342.

8. Lima-Oliveira G, Lippi G, Salvagno GL, Montagnana $M$, Poli G, Solero GP, et al. K(3)EDTA vacuum tubes validation for routine hematological testing. ISRN Hematol 2012;2012:875357. https://doi.org/10.5402/2012/875357.

9. Lombardi G, Ricci C, Banfi G. Effect of winter swimming on haematological parameters. Biochem Med (Zagreb) 2011;21:71-8. https://doi.org/10.11613/BM.2011.014.

10. Favaloro EJ, Lippi G, Adcock DM. Preanalytical and postanalytical variables: the leading causes of diagnostic error in hemostasis? Semin Thromb Hemost 2008;34:612-34. https://doi.org/10.1055/s-0028-1104540. dual anti-platelet therapy. Although AA induced LTA showed a significant bias, the diagnostic accuracy was not influenced at all. Further investigation on healthy subjects without anti-platelet therapy should be performed.

\section{Acknowledgements}

We are grateful to the medical laboratory technicians of the coagulation and the platelet function laboratory, who performed the experimental work.

\section{Potential conflict of interest}

None declared.
11. Lima-Oliveira G, Lippi G, Salvagno GL, Dima F, Brocco $G$, Picheth $G$, et al. Management of preanalytical phase for routine hematological testing: is the pneumatic tube system a source of laboratory variability or an important facility tool? Int J Lab Hematol 2014;36:e37-40. https://doi. org/10.1111/ijlh.12143.

12. Kratz A, Salem RO, Van Cott EM. Effects of a pneumatic tube system on routine and novel hematology and coagulation parameters in healthy volunteers. Arch Pathol Lab Med 2007;131:293-6.

13. Kocak FE, Yöntem M, Yücel O, Cilo M, Genc O, Meral A. The effects of transport by pneumatic tube system on blood cell count, erythrocyte sedimentation and coagulation tests. Biochem Med (Zagreb) 2013;23:206-10. https://doi. org/10.11613/BM.2013.024.

14. Amann G, Zehntner C, Marti F, Colucci G. Effect of acceleration forces during transport through a pneumatic tube system on ROTEM ${ }^{\circledast}$ analysis. Clin Chem Lab Med 2012;50:1335-42. https://doi.org/10.1515/cclm-2011-0800.

15. Colucci G, Giabbani E, Barizzi G, Urwyler N, Alberio L. Laboratory-based $R O T E M\left({ }^{\circledR}\right)$ analysis: implementing pneumatic tube transport and real-time graphic transmission. Int J Lab Hematol 2011;33:441-6. https://doi.org/10.1111/j.1751553X.2011.01303.x.

16. Sandberg S, Fraser CG, Horvath AR, Jansen $R$, Jones $G$, Oosterhuis $W$, et al. Defining analytical performance specifications: Consensus Statement from the 1st Strategic Conference of the European Federation of Clinical Chemistry and Laboratory Medicine. Clin Chem Lab Med 2015;53:833-5. https://doi.org/10.1515/cclm-2015-0067.

17. Medicare, Medicaid and CLIA programs; regulations implementing the Clinical Laboratory Improvement Amendments of 1988 (CLIA)-HCFA. Final rule with comment period. Fed Regist 1992;57:7002-186. 
18. Dyszkiewicz-Korpanty A, Quinton R, Yassine J, Sarode R. The effect of a pneumatic tube transport system on PFA-100 trade mark closure time and whole blood platelet aggregation. J Thromb Haemost 2004;2:354-6. https://doi.org/10.1111/ j.1538-7836.2004.0584e.x.

19. Wallin O, Söderberg J, Grankvist K, Jonsson PA, Hultdin J. Preanalytical effects of pneumatic tube transport on routine haematology, coagulation parameters, platelet function and global coagulation. Clin Chem Lab Med 2008;46:14439. https://doi.org/10.1515/CCLM.2008.288.

20. Bollinger D, Seeberger MD, Tanaka KA, Dell-Kuster S, Gregor M, Zenklusen $U$, et al. Pre-analytical effects of pneumatic tube transport on impedance platelet aggregometry. Platelets 2009;20:458-65. https://doi.org/10.3109/ 09537100903236462.

21. Hübner U, Böckel-Frohnhöfer N, Hummel B, Geisel J. The effect of a pneumatic tube transport system on platelet aggregation using optical aggregometry and the PFA-100. Clin Lab 2010;56:59-64.

22. Glas M, Mauer D, Kassas H, Volk T, Kreuer S. Sample transport by pneumatic tube system alters results of multiple electrode aggregometry but not rotational thrombelastometry. Platelets 2013;24:454-61. https://doi.org/10.3109/09 537104.2012.718383.

23. Thalén S, Forsling I, Eintrei J, Söderblom L, Antovic JP. Pneumatic tube transport affects platelet function measured by multiplate electrode aggregometry. Thromb Res 2013;132:77-80. https://doi.org/10.1016/j.thromres.2013.04.020.
24. Tiwari AK, Pandey P, Dixit S, Raina V. Speed of sample transportation by a pneumatic tube system can influence the degree of hemolysis. Clin Chem Lab Med 2011;50:471-4.

25. Harrison P. Assessment of platelet function in the laboratory. Hämostaseologie 2009;29:25-31.

26. Moffat KA, Ledford-Kraemer MR, Nichols WL, Hayward $C P$; North American Specialized Coagulation Laboratory Association. Variability in clinical laboratory practice in testing for disorders of platelet function: results of two surveys of the North American Specialized Coagulation Laboratory Association. Thromb Haemost 2005; 93:549-53. https://doi. org/10.1160/th04-10-0670.

27. Linnemann B, Schwonberg J, Mani H, Prochnow S, Lindhoff-Last $E$. Standardization of light transmittance aggregometry for monitoring antiplatelet therapy: an adjustment for platelet count is not necessary. J Thromb Haemost 2008;6:677-83. https://doi.org/10.1111/j.15387836.2008.02891.x

28. Cattaneo $M$, Hayward $C P$, Moffat KA, Pugliano MT, Liu $Y$ Michelson AD. Results of a worldwide survey on the assessment of platelet function by light transmission aggregometry: a report from the platelet physiology subcommittee of the SSC of the ISTH. J Thromb Haemost 2009;7:1029. https://doi.org/10.1111/j.1538-7836.2009.03458.x.

29. Cattaneo M, Cerletti C, Harrison P, Hayward CP, Kenny D, Nugent $D$, et al. Recommendations for the standardization of light transmission aggregometry: a consensus of the working party from the platelet physiology subcommittee of SSC/ISTH. J Thromb Haemost 2013;11:1183-89. https:// doi.org/10.1111/jth. 12231 . 